\title{
Epigenetics and Diabetes: Current and Perspective
}

Gouri $\mathrm{A}^{1^{*}}$, Dekaken $\mathrm{A}^{2}$, Chefrour $\mathrm{M}^{3}$, Guieu $\mathrm{R}^{3}$, Benharkat $\mathrm{S}^{1}$

${ }^{1}$ Laboratory of Medical Biochemistry, IBN ROCHD University Hospital, Annaba, Algeria.

${ }^{2}$ Department of Internal Medicine, Pr EL OKBI Public Hospital, Guelma, Algeria.

${ }^{3}$ Laboratory of Biochemistry, Hôpital de La Timone Enfant, Marseille, France.

\begin{abstract}
Diabetes has reached epidemic proportions throughout the world. Increasing evidence suggests that complex interactions between genes and the environment might play a major role in the pathogenesis of this mutlifactoriel disease and its complications, and this might be a result of the involvement of epigenetic factors. Despite the fact that genetic susceptibility and environmental factors have been implicated as key contributors to type1 diabetes (TD1) risk, it is not sufficient to explain the increased incidence of T1D in the world. Epigenetics may explain, at least in part, these phenomena. Recent studies show that epigenetic factors, including DNA methylation and histone modification, may affect the susceptibility for Type 2 diabetes (TD2) and the progression of theirs complications. In this review, we present recent progress in epigenetics mechanisms involved in diabetes development and its most commons complications, and the new approaches of epigenetic modifications as potential new treatment targets in diabetes.
\end{abstract}

Keywords: Epigenetics; Diabetes; DNA Methylation; Histones.

\section{*Corresponding Author:}

Adel Gouri,

Laboratory of Medical Biochemistry, IBN ROCHD University Hospital,

Annaba, Algeria.

Tel: +213666088226

E-mail: pharmagor@gmail.com

Received: April 17, 2015

Accepted: May 29, 2015

Published: June 04, 2015

Citation: Gouri A, Dekaken A, Chefrour M, Guieu R, Benharkat S (2015) Epigenetics and Diabetes: Current and Perspective. Int J Diabetol Vasc Dis Res, 3(5) 94-98. doi: http://dx.doi.org/10.19070/2328-353X-1500019

Copyright: Gouri $\mathbf{A}^{\odot}$ 2015. This is an open-access article distributed under the terms of the Creative Commons Attribution License, which permits unrestricted use, distribution and reproduction in any medium, provided the original author and source are credited.

\section{Introduction}

Diabetes is undoubtedly one of the most challenging health problems in the $21^{\text {st }}$ century.

The estimated worldwide prevalence of diabetes among adults was 366 million in 2011; by 2030 this will have risen to 552 million. Type 2 diabetes is the predominant form and accounts for at least $90 \%$ of cases. $80 \%$ of people with diabetes live in low-and middle-income countries; the greatest numbers of people with diabetes are between 40 to 59 years of age $[1,2]$.

Type 2 diabetes mellitus is a polygenic multifactorial disease characterised by hyperglycaemia and altered lipid metabolism due to impaired insulin secretion from pancreatic $\beta$-cells. Today, it is well established that combinations of non-genetic and genetic risk factors influence the susceptibility for Type 2 diabetes. While obesity, physical inactivity, and aging represent non-genetic risk factors for Type 2 diabetes, genome-wide association studies have identified more than 40 polymorphisms associated with an increased risk for the disease [3-7].

Recent studies show that epigenetic factors, including DNA methylation and histone modification, may affect the susceptibility for Type 2 diabetes [8].

Environmental susceptibility factors also contribute to the risk of developing Type 1 diabetes. From an epigenetic standpoint, the pathologic mechanisms involved in the development of Type 1 diabetes may include DNA methylation, histone modification, microRNA, and molecular mimicry. These mechanisms may act through regulating of gene expression, thereby affecting the immune system response toward islet beta cells [9].

This review will provide recent evidence from the literature supporting the immediate need for further investigation to uncover the power of epigenetics in the prediction, prevention and treatment of Type 1 and 2 diabetes.

\section{Epigenetic Mechanisms}

Epigenetics is presently described as the study of changes in gene expression that occur not by changing the DNA sequence, but by modifying DNA methylation and remodeling chromatin. The major epigenetic mechanisms are DNA methylation, histone modifications and modulation of gene transcription and translation by 
non-coding RNAs, including miRNAs. These epigenetic changes are potentially reversible and modulated by the environment, diet or pharmacological intervention (Figure 1).

DNA methylation is a genomic modification that can influence gene activity. It occurs almost exclusively at the cytosine of $\mathrm{CpG}$ dinucleotides, which tend to cluster in regions called " $\mathrm{CpG}$ islands". The primary function of DNA methylation is to actively silence genes and DNA regions in which transcription is not desired [10].

The modifications of the histones result in conformational changes of the chromatin that alter the access of promoters for transcription factors. These modifications, including acetylation, methylation, phosphorylation, and ubiquitination, alter the interaction between the histones, DNA and nuclear proteins, therefore affecting gene transcription and regulate gene silencing or expression [10].

A third mechanism involves the expression of short noncoding RNAs, whose expression can lead to translational silencing through the specific binding and eventual degradation of transcribed RNA. MicroRNAs (miRNAs) can also regulate DNA methylation and histone modifications [11].

\section{Epigenetic Pathogenesis for Type 1 Diabetes}

Type 1 diabetes (T1D) is a complex autoimmune disease involving the interaction of numerous genes and environmental factors, which may be regulated by epigenetic mechanisms. It results from the immunemediated destruction of the insulin-secreting beta -cells that reside in the pancreatic Islets of Langerhans. A number of linkage and genome-wide association studies have identified the major risk loci for Type 1 diabetes. The DR3, DR4 and DQ2 susceptibility loci are located within the major histocompatibility complex (MHC, also referred to as the human leukocyte antigen, HLA) class II region and can account for more than $\sim 40 \%$ of increased risk $[12,13]$.
Despite the fact that genetic susceptibility and environmental factors have been implicated as key contributors to disease risk, it is not sufficient to explain the increased incidence of T1D in the world. Epigenetics may explain, at least in part, these phenomena.

Epigenetic mechanisms including DNA methylation, post-translational modifications of histones and the activation of microRNAs, could play a role in the initiation or progression of autoimmunity, or alter the target tissue in such a way as to increase the probability that it will be targeted by an autoimmune attack.

T1D results from a $T$ cell-mediated autoimmune attack on pancreatic $\beta$-cells. $\mathrm{CD}^{+} \mathrm{T}$ helper cells are major protagonists involved in $\beta$-cell autoimmunity.

Abnormal global methylation of $\mathrm{CD}^{+}{ }^{+} \mathrm{T}$ cells has been observed in patients with latent autoimmune diabetes in adults compared with healthy control subjects.

Moreover, histones modifications associated with the insulin gene and other developmental regulators required for $\beta$-cell development could modify the maturation or function of $\beta$-cells resulting in predisposition for autoimmune diabetes. Additionally, aberrant gene expression could also result in the targeting of $\mathrm{b}$-cells by the autoimmune response [14].

\section{Epigenetic Pathogenesis for Type 2 Diabetes}

Epigenetic research into of Type 2 diabetes (T2D) is still a very young field. The role of epigenetic mechanisms in the etiology of these disorders and related metabolic abnormalities such as obesity, dyslipidemia, hypertension, and hyperglycemia is not well elucidated. Notably, epigenetic effects may also be affected by the environment, making them potentially important pathogenic mechanisms in complex multifactorial diseases such as Type 2 diabetes (Figure 2). Important evidence for a role of epigenetic factors in the pathogenesis of $\mathrm{T} 2 \mathrm{D}$ comes from a data-mining

Figure 1. Epigenetic mechanisms and gene-activating events in epigenetic pathologies.

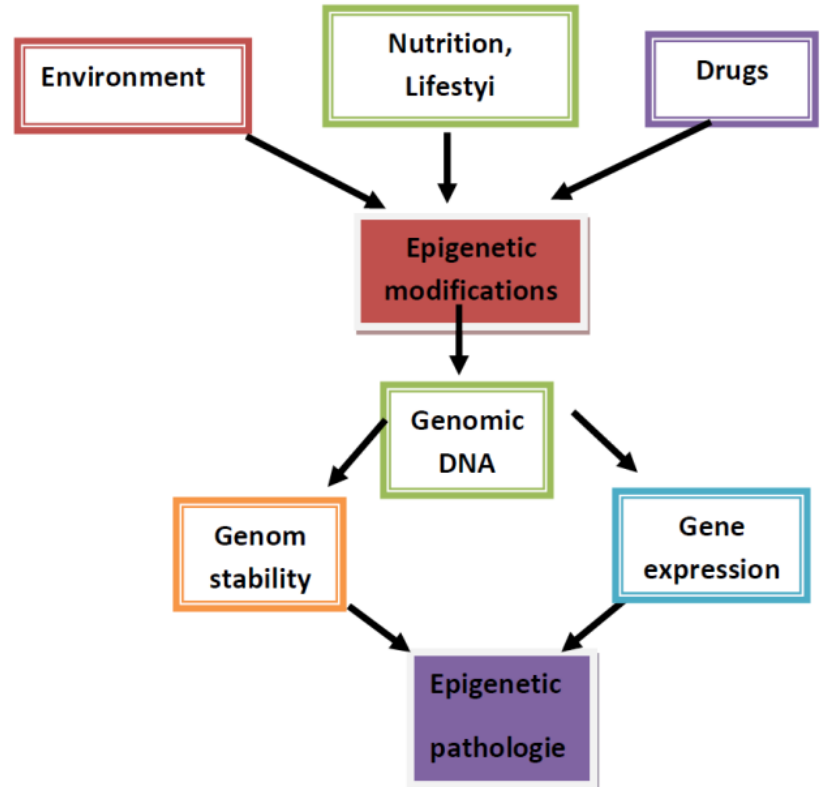


Figure 2. Model proposing a role for epigenetic mechanisms in the pathogenesis of type 2 diabetes.

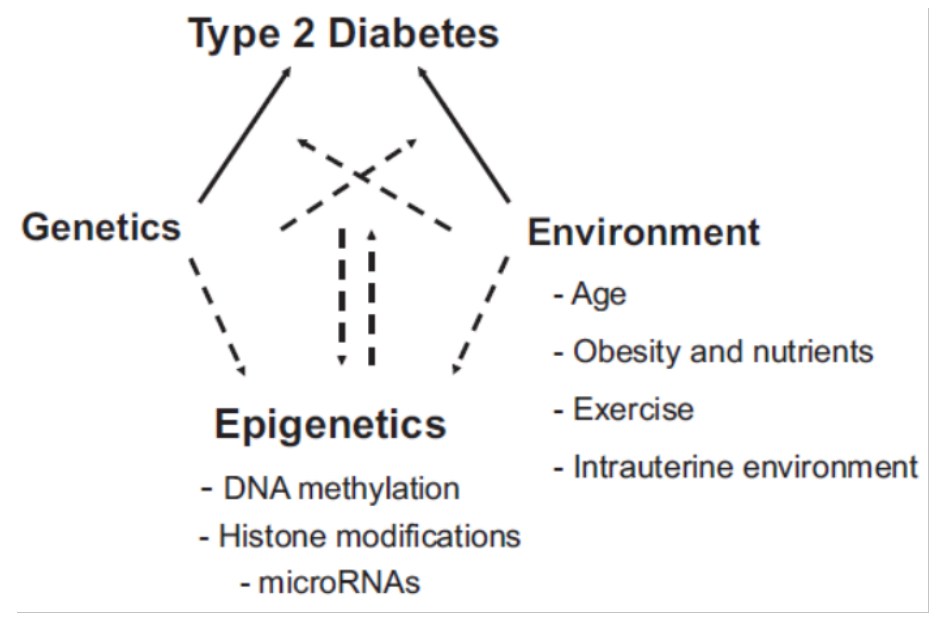

analysis of more than 12 million Medline records [15].

The study found that methylation and chromatin are top hits, implicitly related to T2D.

The epigenetic of T2D is the interaction between gene activation and epidemiology, where gene activation can be in the form of DNA methylation, histone modification or RNA activation. This could be affected by different epidemiological factors, namely age, obesity, nutrition, physical activity and intrauterine environment [16-18].

Epigenetic mechanisms such as DNA methylation and histone modifications are increasingly considered to be important in phenotype transmission and the development of TD2.

In differentiated mammalian cells, the addition of methyl groups to DNA occurs on cytosine residues, and these modifications are mostly established in the context of cytosine guanine dinucleotides (CpGs), a reaction that is carried out by various members of a single family of enzymes. DNA methylation is commonly associated with gene silencing and contributes to $\mathrm{X}$ chromosomal inactivation, genomic imprinting and transcriptional regulation of tissue- specific genes during cellular differentiation [16].

Although data mining analysis has suggested a role for epigenetic factors in the pathogenesis of Type 2 diabetes [19], there are only a limited number of studies that have examined epigenetic changes in target tissues from patients with Type 2 diabetes.

Functional study, evaluating epigenetics in human T2D tissue concerns Peroxisome proliferator-activated receptor gamma coactivator 1 alpha (also known as PGC-1a, and encoded by PPARGC1A), a transcriptional coactivator of mitochondrial genes involved in normal ATP-production and insulin secretion from the pancreatic beta cells, showed that the level of DNA methylation is increased in a promoter region of PPARGC1A in pancreatic islets from patients with T2D, as compared with islets from healthy human donors [20]. Moreover, a global analysis of DNA methylation in skeletal muscle revealed that people with a family history of T2DM have differential DNA methylation of genes involved in muscle function, insulin, and calcium signaling [21].

Furthermore, a link between histone modification and metabo- lism is evident from the observation that loss of histone demethylase (JHDM2A) function leads to obesity and decreased expression of metabolically relevant genes, including peroxisome proliferator-activated receptor alpha (PPARA) and uncoupling protein 1 (UCP1). Similar to DNA methylation, histone modifications also provide a molecular link between a sedentary lifestyle and the development of T2DM [19]. Clearly, the contribution of epigenetic regulation to the manifestation of metabolic disease remains to be completely described.

\section{Epigenetic Modifications and Diabetic Complica- tions}

Diabetes and metabolic disorders are leading causes of microand macrovascular complications such as atherosclerosis, hypertension, nephropathy, retinopathy and neuropathy. One major event in the progression of diabetic complications is vascular inflammation with increased expression of inflammatory genes. Enhanced oxidative stress, dyslipidemia, and hyperglycemia have also been suggested to influence the development of diabetic complications [22].

\section{Cardiovascular complications}

remain the major cause of morbidity and mortality in the diabetic population. It is increasingly appreciated that exposure to high glucose is the major factor leading to these complications. Recent studies have proposed that hyperglycemia may induce epigenetic modifications of genes involved in vascular inflammation.

Such studies have led to the view that the transcriptional determinant, nuclear factor $(\mathrm{NF})-\mathrm{k} B$, which is readily activated by hyperglycemia, plays a pivotal role in diabetic vascular complications [23]. Furthermore, $\mathrm{NF}_{-\mathrm{KB}} \mathrm{B}$ activation leads to the upregulation of molecules such as the chemokine, monocyte chemotactic protein (MCP)-1, and adhesion molecules such as vascular cell adhesion molecule (VCAM)-1, which have been extensively investigated in atherosclerosis [24].

Epigenetic mechanisms such as post translational modification of histones and DNA methylation also play central roles in gene regulation by affecting chromatin structure and function. Recent studies have suggested that hyperglycemia-induced DNA methylation changes that persist into the metabolic memory state. The 
role of DNA methylation in the pathogenesis of cardiovascular diseases (CVDs) is not completely understood.

Atherosclerosis was associated with global hypomethylation in vascular smooth muscle cells (VSMCs) of atherosclerotic lesions from humans [22].

In addition, several studies have implicated miRNAs in diabetes pathogenesis. However, the role of miRNAs in diabetes vascular complications is less studied. Evidence shows that miRNAs can affect the function of both endothelial cells (ECs) and vascular smooth muscle cells (VSMCs) relevant to vascular diseases [25277.

\section{Diabetic nephropathy}

In the diabetic nephropathy (DN), tubulointerstitial fibrosis, due to increased expression of extracellular matrix proteins such as collagens and fibronectins, is initiated and sustained by a number of different factors including the transforming growth factor-beta (TGF $\beta$ ) family. This family of inflammation mediators is documented to be aberrantly expressed in metabolic memory, implicating TGF- $\beta$ as a major mediator of epigenetic events in DN. $[28,29]$

\section{Diabetic retinopathy}

A role for epigenetic mechanism in the pathogenesis of diabetic retinopathy (DR) has been recently proposed. The first of these manuscripts examined the control of VEGF (significant in both the early and late stages of DR) by miR-200b.

The second revealed that the activity of the matrix metalloproteinases MMP2 and MMP9 cause mitochondria DNA (mtDNA) damage and degradation of mitochondrial membranes in retinal capillary cells which in turn induces apoptosis of the same [30].

\section{Epigenetics and Diabetes Treatment}

Significant advances in the treatment of Type 2 diabetes mellitus (T2DM) include the implementation of prevention efforts aimed at delaying progression of glucose intolerance to overt diabetes mellitus (DM) and the development of new classes of blood glucose-lowering medications to supplement existing therapies. While the current management approach for T2DM continues to encompass traditional drugs that focus on $\beta$-cell failure and/ or insulin resistance, newer agents that target other defects (eg, incretin deficiency/resistance) are increasingly incorporated [31].

However, evidence shows that current therapies based on these mechanisms are not fully efficacious in preventing complications, suggesting the need for the identification of novel therapeutic targets. In particular, it has been noted that some individuals with diabetes experience a continued progression of vascular complications even after glycaemic control subsequent to a period of prior hyperglycaemic exposure, a phenomenon termed 'metabolic memory' $[32,33]$.

The studies showed that environment effects can induce epigenetic alterations. These alterations ultimately affect expression of key genes linked to the development of T2DM including genes critical for pancreatic development and $\beta$-cell function, peripheral glucose uptake and insulin resistance. Reversal of epigenetic mechanisms or 'epigenetic therapy' might unveil a critical window during which epigenetic therapeutic agents could be used as means to prevent the later development of a disease.

Three specific epigenetic mechanisms currently under investigation include attempts to silence risk genes by enhancing the methylation of gene promoters or their downstream products; attempts to activate helpful genes by inhibiting an enzyme called histone deacetylase and small RNAs known as microRNAs (miRNAs), which have emerged as a potential target for Type 2 diabetes therapies.

Due to their highly conserved active domain, histone deacetylases (HDACs) have been extensively studied for the development of inhibitors. Most of the currently designed inhibitors fall into four broad classes, short-chain fatty acids (SCFAs), hydroxamates, benzamates, and cyclic tetrapeptides, which for the most part target the class I and class II HDACs [34]. Several inhibitors of the class III HDACs (Sirtuins) have now been synthesized and also show therapeutic potential. Additionally natural prodrugs which target histone deacetylases have also been isolated and include sulforaphane (SFN), diallyl disulfide (DADS), and resveratrol [3539].

Given the emerging body of evidence linking aberrant miRNA expression with diabetes pathogenesis, it is becoming increasingly clear that these ncRNAs may have utility in either the management or treatment of this disease. The possibility of using miRNA and/or siRNA to target diabetes has recently been extensively reviewed [40]. A greater understanding of miRNA target identification/validation, their roles in diabetes pathogenesis, and mechanisms of specific delivery will be required before they can be evaluated as a therapeutic modality.

\section{Conclusion}

Diabetes is multifactorial disease involving interactions between genetic and environmental factors. Alarming estimates indicate that the rates of diabetes and associated complications are rapidly increasing, and therefore additional strategies to curb these trends are needed. Epigenetics provides a mechanism which may explain the etiology of diabetes and the diversity of phenotypes in the general population.

Epigenetics provides a mechanism which may explain the etiology of diabetes and the diversity of phenotypes in the general population.

Although there is support for the role for epigenetics in the pathogenesis of diabetes and its complications, conclusive studies from human diabetes tissues are limited.

The perspective of epigenetic control is slowly growing from the view that genomic imprints are irreversibly fixed to the notion that epigenetic DNA modifications can be rapid, reversible, and responsive to both environmental and lifestyle inputs, it may thus be possible to test epigenetic drugs as putative novel drugs for the treatment of diabetes and its complications.

\section{References}

[1]. Chen L, Magliano DJ, Zimmet PZ (2011) The worldwide epidemiology of type 2 diabetes mellitus--present and future perspectives. Nat Rev Endo- 
crinol 8(4): 228-36.

[2]. Nolan CJ, Damm P, Prentki M (2011) type 2 diabetes across generations: from pathophysiology to prevention and management. Lancet 378(9786): 169-81.

[3]. Saxena R, Voight BF, Lyssenko V, Burtt NP, de Bakker PI, et al., (2007) Genome-wide association analysis identifies loci for type 2 diabetes and triglyceride levels. Science 316(5829): 1331-1336.

[4]. Scott LJ, Mohlke KL, Bonnycastle LL, Willer CJ, Li Y, et al., (2007) genome-wide association study of type 2 diabetes in Finns detects multiple susceptibility variants. Science 316(5829): 1341-1345

[5]. Sladek R, Rocheleau G, Rung J, Dina C, Shen L, et al., (2007) A genomewide association study identifies novel risk loci for type 2 diabetes. Nature 445(7130): 881-885.

[6]. Zeggini E, Weedon MN, Lindgren CM, Frayling TM, Elliott KS, et al., (2007) Wellcome Trust Case Control Consortium (WTCCC), Replication of genome-wide association signals in UK samples reveals risk loci for type 2 diabetes. Science 316(5829): 1336-134.

[7]. Zeggini E, Scott LJ, Saxena R, Voight BF, Marchini JL, et al., (2008) Metaanalysis of genome-wide association data and large-scale replication identifies additional susceptibility loci for type 2 diabetes. Nat Genet 40(5): 638-645.

[8]. Ling C, Groop L (2009) Epigenetics: a molecular link between environmental factors and type 2 diabetes. Diabetes 58(12): 2718-25.

[9]. Xie Z, Chang C, Zhou Z (2014) Molecular mechanisms in autoimmune type 1 diabetes: a critical review. Clin Rev Allergy Immunol. 47(2): 174-92

[10]. Waki H, Yamauchi T, Kadowaki T (2012) The epigenome and its role in diabetes. Curr Diab Rep 12(6):673-85.

[11]. Magklara A, Lomvardas S (2012) Epigenetics and Human Disease. N. Ahituv (1st edtn), Gene Regulatory Sequences and Human Disease 253279.

[12]. Bluestone JA, Herold K, Eisenbarth G (2010) Genetics, pathogenesis and clinical interventions in type 1 diabetes. Nature 464(7293): 1293-300

[13]. Ziegler AG, Nepom G (2010) Prediction and pathogenesis in type 1 diabetes. Immunity 32(4): 468-478.

[14]. MacFarlane AJ (2012) Epigenetic Epidemiology of Type 1 Diabetes. K.B. Michels (1st edtn), Epigenetic Epidemiolog 377-400.

[15]. Wren JD, Garner HR (2005) Data-mining analysis suggests an epigenetic pathogenesis for type 2 diabetes. J Biomed Biotechnol (2): 104-112.

[16]. Villeneuve LM, Reddy MA, Natarajan R (2011) Epigenetics: deciphering its role in diabetes and its chronic complications. Clin Exp Pharmacol Physiol 38(7): 451-9.

[17]. Pinney SE, Simmons RA (2010) Epigenetic mechanisms in the development of type 2 diabetes. Trends Endocrinol Metab 21(4): 223-9.

[18]. Ling C, Groop L (2009) Epigenetics: A Molecular Link Between Environmental Factors and Type 2 Diabetes Diabetes 58(12): 2718-25.

[19]. Kirchner H, Osler ME, Krook A, Zierath JR (2013) Epigenetic flexibility in metabolic regulation: disease cause and prevention?. Trends Cell Biol 23(5): 203-9

[20]. Lin J, Handschin C, Spiegelman BM (2005) Metabolic control through the PGC-1 family of transcription coactivators. Cell Metab1(6): 361-370.

[21]. Nitert MD (2012) Impact of an exercise intervention on DNA methylation in skeletal muscle from first-degree relatives of patients with type 2 diabetes.
Diabetes 61(12): 3322-3332.

[22]. Reddy MA, Natarajan R (2011) Epigenetic mechanisms in diabetic vascular complications. Cardiovasc Res 90(3): 421-9.

23]. Brownlee M (2001) Biochemistry and molecular cell biology of diabetic complications. Nature 414(6865): 813-820.

[24]. Burke AP, Kolodgie FD, Zieske A, Fowler DR, Weber DK, et al., (2004) Morphologic findings of coronary atherosclerotic plaques in diabetics: a postmortem study. Arterioscler Thromb Vasc Biol 24(7): 1266-1271.

[25]. Urbich C, Kuehbacher A, Dimmeler S (2008) Role of microRNAs in vascular diseases, inflammation, and angiogenesis. Cardiovasc Res 79(4): 581588.

[26]. Muhonen P, Holthofer H (2009) Epigenetic and microRNA-mediated regulation in diabetes. Nephrol Dial Transplant 24(4): 1088-1096.

[27]. Cordes KR, Srivastava D (2009) MicroRNA regulation of cardiovascular development. Circ Res 104(6): 724-732.

[28]. Burns WC, Twigg SM, Forbes JM, Pete J, Tikellis C, et al., (2006) Connective tissue growth factor plays an important role in advanced glycation end product-induced tubular epithelialto- mesenchymal transition: implications for diabetic renal disease. J Am Soc Nephrol 17(4): 2484-94.

[29]. Wang B, Koh P, Winbanks C, Coughlan MT, McClelland A, et al., (2011) miR-200a Prevents renal fibrogenesis through repression of TGF-beta2 expression. Diabetes 60(1): 280-7.

[30]. Zhong Q, Kowluru RA (2013) Regulation of matrix metalloproteinase-9 by epigenetic modifications and the development of diabetic retinopathy. Diabetes 62(7): 2559-68.

[31]. Mazzola N (2012) Review of Current and Emerging Therapies in Type 2 Diabetes Mellitus . Am J Manag Care 18(1): 17-26.

[32]. Gray SG (2012) The Potential of Epigenetic Compounds in Treating Diabetes. T. Tollefsbol (1st edtn), Epigenetics in Human Disease.

[33]. Nuria C, Bramswig, Klaus H, Kaestner (2012) Epigenetics and diabetes treatment: an unrealized promise? Trends Endocrinol Metab 23(6): 286-91.

[34]. Bolden JE, Peart MJ, Johnstone RW (2006) Anticancer activities of histone deacetylase inhibitors. Nat Rev Drug Discov 5(9): 769-784.

[35]. Outeiro TF, Kontopoulos E, Altman S, Kufareva I, Strathearn KE, et al., (2007) Sirtuin 2 Inhibitors Rescue a-Synuclein-Mediated Toxicity in Models of Parkinson's Disease. Science 317(5837): 516-519.

[36]. Schuetz A, Min J, Antoshenko T, Wang CL, Allali-Hassani A, et al., (2007) Structural basis of inhibition of the human NADp-dependent deacetylase SIRT5 by suramin. Structure 15(3): 377-389.

[37]. Kiviranta PH, Leppanen J, Kyrylenko S, Salo HS, Lahtela-Kakkonen M, et al., (2006) N, N'-Bisbenzylidenebenzene- 1,4-diamines and N, N'-Bisbenzylidenenaphthalene-1,4-diamines as Sirtuin Type 2 (SIRT2). Inhibitors. J Med Chem 49(26): 7907-11.

[38]. Tervo AJ, Suuronen T, Kyrylenko S, Kuusisto E, Kiviranta PH, et al., (2006) Discovering inhibitors of human sirtuin type 2: novel structural scaffolds. J Med Chem 49(24): 7239-41.

[39]. Dashwood RH, Ho E (2007) Dietary histone deacetylase inhibitors: From cells to mice to man. Semin Cancer Biol 17(5): 363-9.

[40]. Czech MP, Aouadi M, Tesz GJ (2011) RNAi-based therapeutic strategies for metabolic disease. Nat Rev Endocrinol 7(8): 473-484. 